\title{
A Case of the Vibrant Soundbridge Stapes Coupler in Patients with Mixed Hearing Loss
}

\author{
Ah Young Park ${ }^{1}$, Ju Hyun Jeon ${ }^{2}$, In Seok Moon ${ }^{1}$, and Jae Young Choi ${ }^{1}$ \\ ${ }^{1}$ Department of Otorhinolaryngology, Yonsei University College of Medicine, Seoul, \\ ${ }^{2}$ Department of Otorhinolaryngology, Inje University College of Medicine, Busan, Korea
}

$\begin{array}{ll}\text { Received } & \text { May 24, } 2014 \\ \text { Revised } & \text { July 23, } 2014\end{array}$

Accepted August 23, 2014

Address for correspondence

In Seok Moon, MD, PhD

Department of Otorhinolaryngology,

Yonsei University

College of Medicine,

50-1 Yonsei-ro, Seodaemun-gu,

Seoul 120-752, Korea

Tel $+82-2-2228-3627$

Fax $+82-2-393-0580$

E-mailismoonmd@yuhs.ac
The Vibrant Soundbridge (VSB) with stapes clip coupler placement at the stapes head has been used successfully to treat mixed hearing loss. Coupling between the floating mass transducer of the VSB and the stapes head is technically less demanding than incus vibroplasty and is more likely to generate a positive outcome without significantly changing residual hearing or resulting in medical or surgical complications. A 65-year-old man with bilateral mixed hearing loss and chronic otitis media underwent vibroplasty with a stapes clip coupler. Speech discrimination scores in both quiet and noise environments showed better functional gain with the VSB than with the use of a conventional hearing aid. The results of the present case show the feasibility of implanting a VSB with a stapes coupler in patients with mixed hearing loss due to chronic otitis media.

Korean J Audiol 2014;18(2):93-96

\section{Introduction}

Active middle ear implants are an alternative treatment option for various forms of hearing loss. The classic indication for the use of the Vibrant Soundbridge (VSB, MED-EL, Innsbruck, Austria) is sensorineural hearing loss, in which case the floating mass transducer (FMT) is crimped to the long process of the incus with a titanium clip. ${ }^{1)}$ When used to treat mixed hearing loss, in which the middle ear is diseased and the inner ear requires amplified auditory input, the FMT can also be used together with various titanium couplers to ease the attachment of the FMT to the ossicles of the middle ear or to the oval or round window, thereby stimulating the inner ear. Furthermore, the FMT in conjunction with a coupling element can be used not only as an active prosthesis but also works as a passive prosthesis without activation of the speech processor. ${ }^{2)}$

In 2006, a Bell partial prosthesis in combination with an FMT was tested in three temporal bones. ${ }^{3)}$ Although the results of laser Doppler interferometry were encouraging, the lateral

This is an Open Access article distributed under the terms of the Creative Commons Attribution Non-Commercial License (http://creativecommons. org/licenses/by-nc/3.0/) which permits unrestricted non-commercial use, distribution, and reproduction in any medium, provided the original work is properly cited. attachment of the prosthetic shaft to the FMT runs the risk of prosthesis tilting; thus, this Bell vibroplasty technique has not been widely performed. In 2011, the concept was improved by the development of Clip-PORP-Vibroplasty. ${ }^{4)}$ The coupler combines a titanium clip holder for the FMT with the classic mechanism that involves fitting the clip onto the stapes head in a standardized way, as with passive partial clip prostheses. ${ }^{5)}$ This linear integration of the FMT in a titanium column with additional fastening by a clip mechanism adds potential stability and long-term safety to the construction. This coupling element is also available with a bell-shaped attachment (VibroplastyBell-Coupler) to the stapes head.

We report herein a case involving a patient with VSB who underwent stapes clip coupler placement at the stapes head.

\section{Case Report}

A 65-year-old man with postlingual-onset hearing loss was diagnosed with bilateral mixed severe hearing loss due to chronic otitis media. He had used conventional hearing aids in both ears for more than 3 years, but he no longer used his right hearing aid because of discomfort. He experienced difficulty in using the telephone. 

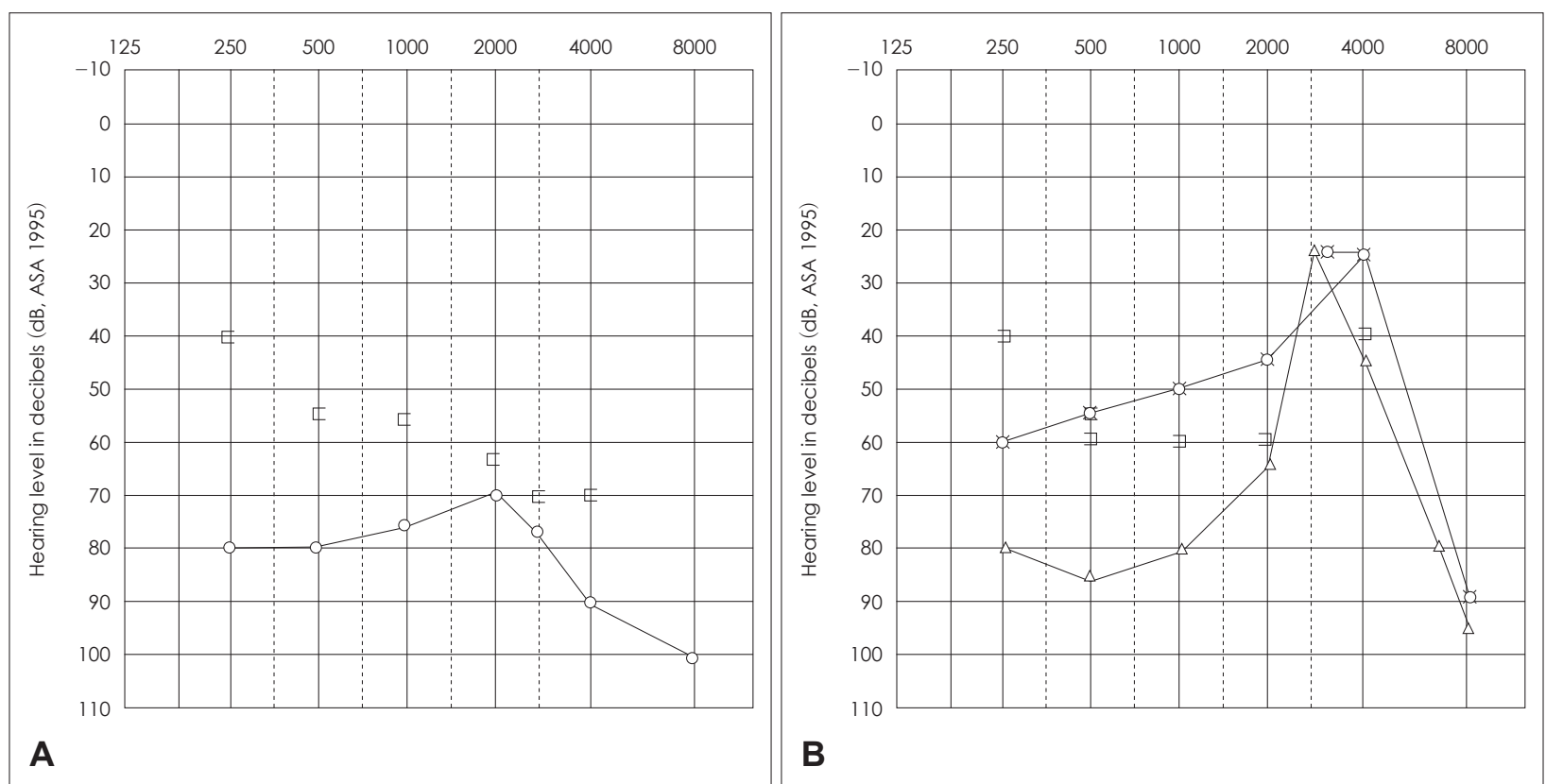

Fig. 1. A: Pure tone audiometry (PTA) for the right ear shows moderate-to-severe mixed hearing loss. B: PTA for the left ear also shows moderate-to-severe mixed hearing loss and aided PTA shows $50 \mathrm{~dB}$.

Otoscopic examination revealed sclerotic tympanic membranes without perforation or effusion. A pure tone audiogram revealed bilateral symmetrical mixed hearing loss with an average air conduction threshold of $78 \mathrm{~dB}$ in the right ear and 69 $\mathrm{dB}$ in the left (Fig. 1). Speech discrimination scores were $62 \%$ for the right ear at $104 \mathrm{~dB}$ most comfortable listening (MCL) and $88 \%$ for the left ear at $98 \mathrm{~dB}$ MCL without hearing aids. He used the left-sided conventional hearing aid only. In the left ear, aided pure-tone audiometry showed $50 \mathrm{~dB}$ and aided speech audiometry showed $86 \%$ at $70 \mathrm{~dB}$ MCL (Fig. 1B). Sclerotic changes in the mastoid cavity, a normal ossicular chain, and soft tissue filling the middle ear cavities were noted on preoperative temporal bone CT scans. The patient was suspected to have bilateral chronic otitis media (Fig. 2).

We planned a right mastoidectomy to control the inflammation, and placement of a right middle ear implant to improve hearing. During the operation, we first performed a simple mastoidectomy to remove sclerotic mastoid air cells and granulation, then widely opened the facial recess. Although soft tissue around the ossicular chain was removed, incudostapedial joint fixation noted. The incudostapedial joint was separated to confirm stapes mobility. Because stapes mobility was intact, incus was then removed and the VSB internal device (MEDEL; Innsbruck, Austria) was implanted. After cutting the attachment clip of the FMT with surgical knife (Fig. 3A), the FMT was inserted into a clip coupler (Fig. 3B). The coalescent clip coupler-FMT complex (Fig. 3C) was connected to the intact head of the stapes (Fig. 3D). Fixation between the clip coupler-
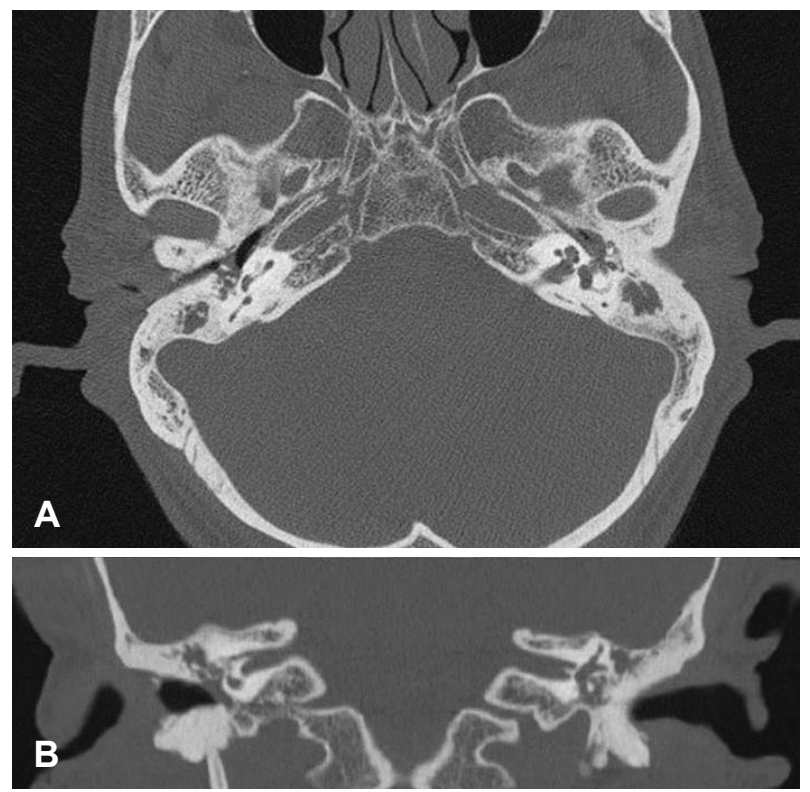

Fig. 2. Axial (A) and coronal (B) view of temporal bone computed tomography scan shows sclerotic change of the mastoid cavity, normal ossicular chain, and soft tissue filling middle ear cavities suspicious for bilateral chronic otitis media.

FMT complex and stapes head was stabilized by clipping (Fig. $3 \mathrm{E})$. Harvested conchal cartilage was inserted between the FMT and tympanic membrane to prevent protrusion of the clip coupler-FMT complex. The patient was discharged on the second day postoperatively without complications. Six months after the operation, the patient showed a decreased air-bone gap on mid frequencies even without the speech processor 
Fig. 3. After cutting the attachment clip with surgical knife $(A)$, floating mass transducer (FMT) was inserted into clip-coupler (B). The coalescent clipcoupler-FMT complex (C) was connected to the intact head of stapes and fixed by clipping (black arrow)(D). We ascertained stabilization status between clip-coupler-FMT complex and stapes head (white arrow)(E).

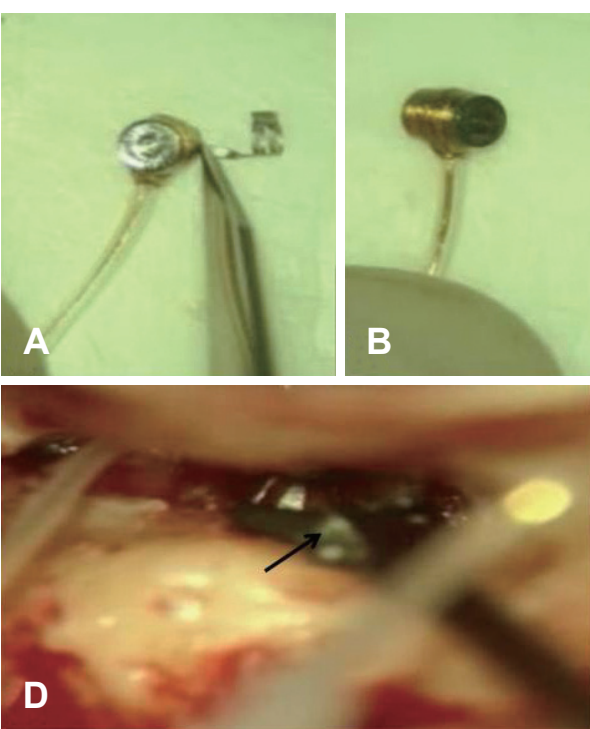

D

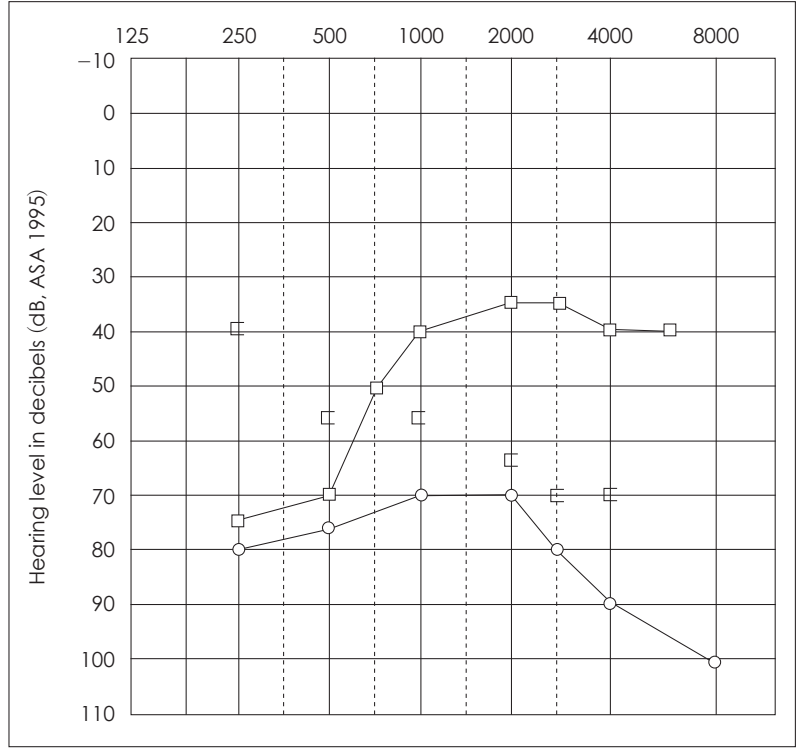

Fig. 4. Six weeks after the surgery, both decreased air-bone gap with and without the speech processor was noted on pure tone audiometry. High-tone frequency hearing was significantly improved on activation of the speech processor.

( $>5 \mathrm{~dB}$ decrease on $0.5 \& 1.0 \mathrm{kHz}$ ). Additionally, on activation of the speech processor, vibroplasty can actively improve mid- and high-tone frequency hearing and patients showed more efficient sound transmission (Fig. 4).

The Abbreviated Profile of Hearing Aid Benefit (APHAB) questionnaire, which assesses the performance of, and satisfaction with, hearing aids, was administered before and after the operation. The APHAB score showed marked improvement from 76.0 points preoperatively to 12.8 points postoperatively (ease of communication, 0 ; reverberation, 17; background noise, 17; aversiveness, 17).

\section{Discussion}

Originally, the VSB was designed for the treatment of sensorineural hearing loss in normal middle ears. With the clip holder, it can now also be used in pathologic middle ears with a stable and reliable attachment. Previous experiments have shown that inner ear stimulation is more effective than conventional hearing aids and passive middle ear implants in pathologic middle ear conditions.

The hearing outcome in our case was more satisfactory than those in previous reports of partial ossicular reconstruction prosthesis coupling on the stapes head. Efficient sound transmission was clinically confirmed according to the temporal bone experiments. ${ }^{3)}$

Our patient reported very comfortable hearing even without the use of a speech processor. Upon application of the speech processor, the patient exhibited a marked improvement in the hearing threshold and great comfort of use, which were shown by the results of the APHAB Questionnaire. ${ }^{6,7)}$ These positive outcomes can be explained by involvement of both passive and active pathways. Use of the clip vibroplasty device alone can produce passive PORP effects by adjust the thickness of reinforcement cartilage. Additionally, on activation of the speech processor, vibroplasty can actively improve high-tone frequency hearing and speech discrimination without the occlusion effect. $^{8,9)}$

Clip vibroplasty is recommended only after complete eradication of any infectious disease of the ear in patients with chronic otitis media, and the cable of the implant should be protected behind thick cartilage plates. Two step approaches for clip vibroplasty should be considered if any infectious condition is remained. 
Because of the improvement in hearing and ease of handling, similar to the placement of a passive prosthesis, the indications for this power clip may make it a promising alternative for patients with conductive hearing loss who got poor results from passive middle ear implants (ossiculoplasty).

\section{Acknowledgments}

This study was supported by a grant from the Korea Health Technology R\&D Project, Ministry of Health \& Welfare, Republic of Korea (A102065).

\section{REFERENCES}

1) Dazert S, Shehata-Dieler WE, Dieler R, Helms J. ["Vibrant Soundbridge" middle ear implant for auditory rehabilitation in sensory hearing loss. I. Clinical aspects, indications and initial results]. Laryngorhinootologie 2000;79:459-64.

2) Zahnert T, Bornitz M, Hüttenbrink KB. Experiments on the coupling of an active middle ear implant to the stapes footplate. Adv Otorhinolaryngol 2010;69:32-7.

3) Huber AM, Ball GR, Veraguth D, Dillier N, Bodmer D, Sequeira D.
A new implantable middle ear hearing device for mixed hearing loss: a feasibility study in human temporal bones. Otol Neurotol 2006;27: 1104-9.

4) Hüttenbrink KB, Beutner D, Bornitz M, Luers JC, Zahnert T. Clip vibroplasty: experimental evaluation and first clinical results. Otol Neurotol 2011;32:650-3.

5) Hüttenbrink KB, Zahnert T, Wüstenberg EG, Hofmann G. Titanium clip prosthesis. Otol Neurotol 2004;25:436-42.

6) Zwartenkot JW, Hashemi J, Cremers CW, Mulder JJ, Snik AF. Active middle ear implantation for patients with sensorineural hearing loss and external otitis: long-term outcome in patient satisfaction. Otol Neurotol 2013;34:855-61.

7) Garin P, Schmerber S, Magnan J, Truy E, Uziel A, Triglia JM, et al. Bilateral vibrant soundbridge implantation: audiologic and subjective benefits in quiet and noisy environments. Acta Otolaryngol 2010; 130:1370-8.

8) de Abajo J, Sanhueza I, Giron L, Manrique M. Experience with the active middle ear implant in patients with moderate-to-severe mixed hearing loss: indications and results. Otol Neurotol 2013;34:1373-9.

9) Beleites T, Neudert M, Beutner D, Hüttenbrink KB, Zahnert T. Experience with vibroplasty couplers at the stapes head and footplate. Otol Neurotol 2011;32:1468-72. 